\title{
Right Pulmonary Artery
}

National Cancer Institute

\section{Source}

National Cancer Institute. Right Pulmonary Artery. NCI Thesaurus. Code C33489.

A blood vessel arising from the bifurcation of the pulmonary artery that carries

deoxygenated blood to the right lung. 